\title{
Automatic Fall Detection using Smartphone Acceleration Sensor
}

\author{
Tran Tri Dang \\ Falculty of Computer Science and \\ Engineering \\ Ho Chi Minh City University of \\ Technology, VNU-HCM \\ Ho Chi Minh city, Vietnam
}

\author{
Hai Truong \\ Falculty of Computer Science and \\ Engineering \\ Ho Chi Minh City University of \\ Technology, VNU-HCM \\ Ho Chi Minh city, Vietnam
}

\author{
Tran Khanh Dang \\ Falculty of Computer Science and \\ Engineering \\ Ho Chi Minh City University of \\ Technology, VNU-HCM \\ Ho Chi Minh city, Vietnam
}

\begin{abstract}
In this paper, we describe our work on developing an automatic fall detection technique using smart phone. Fall is detected based on analyzing acceleration patterns generated during various activities. An additional long lie detection algorithm is used to improve fall detection rate while keeping false positive rate at an acceptable value. An application prototype is implemented on Android operating system and is used to evaluate the proposed technique performance. Experiment results show the potential of using this app for fall detection. However, more realistic experiment setting is needed to make this technique suitable for use in real life situations.
\end{abstract}

Keywords-fall detection; long lie detection; acceleration sensor; smartphone; personal healthcare

\section{INTRODUCTION}

The proportion of old people in the world population is increasing. According to a report prepared by the Population Division of the United Nations, this number is projected to reach 21 percent in 2050, although it was only 10 percent in 2000 [1]. Translating these ratios into absolute values, there are approximately 600 million elderly people at the start of the twenty-first century, and 50 years later the number of people whose ages are 60 or more will be around 2 billion. The high number of older people brings challenges for the healthcare system, especially at developing countries, where public healthcare service is of limited and expensive.

One of the most popular problems elderly people face is falling. According to the Centers for Disease Control and Prevention, one out of three older people falls each year [2]. The consequences of falls are serious and include: broken bones, head injury [3][4], traumatic brain injuries [5]. If prevention solutions are not invested in the immediate future, the number of injuries caused by falls will be double in 2030 due to the increasing portion of old people [6].

The definition of fall is very common, however, it is difficult to precisely describe a fallen behavior, and thus, to specify the means of detection. In 1987, Gibson [7] defines a fall as "unintentionally coming to ground, or some lower level not as a consequence of sustaining a violent blow, loss of consciousness, sudden onset of paralysis as in stroke or an epileptic seizure", or in an informal explanation, a fall could be fast changes of upstanding/sitting posture to the lolling position without being controlled or without intentional behavior such as lying down. The Gibson 's definition is widely applied in many research aspects because it is broad enough to even cover specific falls caused by syncope or dizziness which could be a consequence of an epileptic fit or cardiovascular collapses. A fall detection system must be able to classify, or distinguish between a fall event with normal behavior to decrease the false positive alarm bothering the elderly people. Concurrently, this system possesses the ability of covering all fall for safety requirement. As a result, how to design a detection system which can balance these two requirements is a challenging mission.

A fall detection system is first designed not to reduce the occurrence of fallen but aims to alert when a fall event happens. However, fall detectors have been demonstrated to direct impact on the reduction of fall fear. In fact, falls and fear of falling is not independent. An individual who is frequently falls appears to be fear of falling and this fear afterwards may increase the risk of suffering from a fall [8]. Fear of fall majorly negative impacts on the life quality of elderly which can cause depression, activities limitation, social interaction decreasing, falling, lower life quality. The relationship between automatic fall detection system and fall fear has been proved by Brownsel [9] et al. They conducted a study on elderly who experienced at least one fall in the previous six months. At the end of the experiment, people who wore the fall detector feel more confident and diminish the fear of fallen, as well as consider the detector had improved their safety.

The other important objective of a fall detector is to limit the time the elderly remains on the floor after falling. The period of laying on the floor after falling determines the severity of a fall because long lie may lead to hypothermia, dehydration and pressure sores [10,11]. This is extremely critical in case the person lives alone without any assistances from their families and neighbors. Lord et al. [12] indicates that about $20 \%$ of fallen patients admitted in the hospital after laying on the ground for more than one hour. Although there is no direct injury at the fallen time, the morbidity rates are very high compared with the patients who entered the hospital in less than 30 minutes. The ultimate goal of the detector system is to realize a fall event and manage to notify an assistant immediately. A robust fall detector should be able to classify the falls as falls and the non-falls as non-falls in real life condition because people sometimes intentionally up stand or sit rapidly, which could confuse the system. Certainly, if an elderly falls and the system is unable to detect, the outcome 
could be dramatic. In addition, people is losing confidence in the detector system, which leads to increase the fear of falling and fallen probability consequently. On the other hand, an overestimated detector system may alert excessive number of false activations, thus, caregivers may assume it as ineffective or useless. Balancing these two objectives is a challenging objective and although several commercial products are available on the market, they are not truly impact on the elders' lives yet $[13,14]$.

\section{RELATED WORKS}

Based on whether the system is attaching on the customer's body or not, the detector could be classified as context-aware systems and wearable devices.

The context-aware systems deploy sensors in the environment to recognize a fall event. One of the drawback of this approach is that fallen could be only alerted limited in places attached with sensors. By contrast, person does not need to wear any special devices which emit many electromagnetic radiations. The most common sensors are cameras, microphones, floor sensors, infrared sensors and pressure sensors. Based on the specific sensor type, detection techniques vary a lot. Most of them utilize the common approach of extracting the personal features and comparing with the model to determine a fallen event. Features which could be collected are the ratio of weight and height [15], changes in light and illumination [16], direction of main axis of the body [17], skin color to detect the body region [18]. These features are then analyzed to distinguish between normal behaviors and fall events by different techniques. For example, Hazelhoff [19] et al. first performs the object segmentation based on the background subtraction and then tracks the object by its motion and head region. Finally, a multi-frame Gaussian classifier is utilized to determine a fall event. Liu et al. [20] use the frame differencing approach to identify the human body. Then, image processing techniques are applied to smooth the input. The authors use k-nearest neighbor classifier to categorize the body posture and a fall is decided based on the time difference of event transitions. Several other approaches are also employed such as Rule-based techniques [21], Bayesian filtering [22], Hidden Markov Models [23], Threshold techniques [24] and Fuzzy Logic [25]. Among these decision and extraction techniques, none of them shows outstanding performance to the others and no appropriate comparison has been done yet. As a result, there is no standardized context-aware technique which is widely accepted by the research community.

Wearable device is defined as electronic sensors which must be worn by the user under, with or on top of clothing. About $90 \%$ of these systems are in the form of accelerometer devices. Some of them also integrate with gyroscopes to extract information about the position of the patient. This trend is rapidly developing due to the cheap embedded sensors. The wearable devices could further be divided into two groups which are accelerometers attached to the body and smartphone built-in accelerometer.

For the accelerometer attached to the body, data is continuously collected during normal activities and falls using independent tri-axial accelerometers attached to different parts of the body. Doukas et al. [26] applied the sensor to the patient's foot in order to transmit patient movement data wirelessly to the monitoring center. The center generalizes data in the three axis and uses machine learning method to classify an event a fall or not. The experiment of this research achieved high sensitivity (SE) and specificity (SP) at SE equals $98.2 \%$ and SP equals $96.7 \%$. The system is also enhanced by transmitting video images for remote decision for any suspected, indecisive falls. However, the authors just perform the experiment on 1 subject, thus, the result is not truly supportive. In another research, Cheng et al. [27] tried to monitor daily activity and fall detection by using sensors attached in the chest and thigh. For the decision, the authors used a decision tree which was constructed on the body posture angels to recognize posture transition and the impact magnitude is thresholded to detect falls. The system is able to detect four different fallen types: from standing to face-up lying, face-down lying, left-side lying and right-side lying. The experiments are performed on 6 males and 4 females from age 22-26 and achieve SE equals $95.33 \%$ and SP equals $97.66 \%$. Most of the existing researches apply thresholding techniques for automatic fall detection. However, since 2010, machine learning approach has increasingly influenced in this area. These methods include Support Vector Machine, Gaussian Distribution of Cluster Knowledge [28] and Decision tree. Among them, multilayer perceptron seems to prevail although standardized technique still has not widely accepted [29]. In term of sensor placement, waist seems to be optimal because it is close to the center of body gravity, supporting reliable information on patient body movements.

Another direction of the wearable devices is smartphone built-in accelerometer. The advantage of this approach is today's smartphones embeds multiple sensors such as camera, microphone, GPS, accelerometer, digital compass and gyroscope. Sposaro et at. [30] proposed an alert system using smartphone which is assume to hold at the thigh (pocket). This system uses thresholding to consider the impact, the difference in position before and after the fall. This approach is also applied in other studies [31,32]. Similar to the attached accelerometer mentioned in previous part, machine learning methods such as support vector machines, Sparse Multinomial Logistic Regression are used to classify a fallen event on the mobilephone. Regarding the position and direction of the phone, waist is still the preferred part of the body. Some of these studies published their result into application which are available for downloading in Google Play. However, when searching with the keyword "fall detector" or "fall detection", 10 results are returned. The number of download is quite low and an average of about 10 people give comments about their opinion which can infer that these application does not attract people much. Therefore, studies in this approach of using smartphone should be invested more because it is anticipated to be an emerging field in the near future.

\section{OUR FALL DETECTION APPROACH}

In this work, our main objective is to determine whether a person is doing her daily activities (e.g. walking, sitting, standing, etc.) or just falls to the ground. We observe that the body of the target person moves significantly differently between theses 2 situations. As a result, if we attach a smart phone to a fixed position of the person body, and make that 
smart phone record the body movements, recorded data could be analyzed to spot the differences between 2 situations. By recognizing the movement differences, we can deduce when a fall happens.

When a body is moving, many types of data are generated. Among them, acceleration is considered as one of the most suitable data type for the purpose of fall detection. The reason behind this consideration is that a body acceleration maintains a strong relationship with the force exerting on that body, according to physical laws, and when there is a fall, the exerting force will be changed accordingly. This transitive relationship between a body fall and its acceleration explains for the reason to apply acceleration data for fall detection. Furthermore, accelerometer is a popular sensor type. It is equipped on most smart phones nowadays. Therefore, using acceleration as the data source to detect fall can make our solution reach a large number of users.

To propose an appropriate algorithm for fall detection, at first, the patterns of acceleration data generated in a fall as well as in other normal activities must be discovered. This helps us in designing an effective classification algorithm and in determining the classification parameters for example the number of parameters and relationships among them. To achieve this objective, data acquisition and analysis phase are executed, which will be described in detailed later. Data is acquired by volunteers who perform intentional activities. Then, acquired data values are visualized to learn the attributes and patterns.

\section{A. Data acquisition}

To acquire acceleration data, a simple Android app is created to read the values provided by a smart phone's accelerometer. A person is invited to use this smart phone to generate data. Because at this stage, we only want to understand the generic different patterns generated during various activities, so one individual is enough to collect the desired data. The smart phone is assumed to be kept at a fixed position on the individual's body. In this research, the individual's front pant pocket is chosen as the position of the smart phone because people frequently keep their phones at that place. In addition, putting a smart phone in pant pocket helps them to keep the phone longer without tiredness.

We ask a volunteer to do the following actions: sitting on a chair, walking, standing up, sitting down, and falling onto a soft mattress, all with a monitoring smart phone in his front pant pocket. The generated acceleration data is processed as follow:

- When there is a change in acceleration value, Android raises an event and also supplies current value in 3 coordinate axes $\left(A_{x}, A_{y}, A_{z}\right)$.

- The amplitude of the current acceleration is calculated as:

$$
A=\sqrt{A_{x}^{2}+A_{y}^{2}+A_{z}^{2}}
$$

\section{B. Data analysis}

For each activity, the acceleration amplitudes are stored as time series. Microsoft Excel is utilized to plot these series to see the pattern of various activities. Figure 2. shows the results of the plotting.

Based on the illustration images of acceleration data in various cases, some important features are indicated:

- In sitting state, acceleration value is quite stable. It is easy to understand because the experiment smart phone is almost static in sitting state.

- In walking state, acceleration value falls down, rises up, then repeats this cycle again. The difference between minimum and maximum value in a cycle is about $2 \mathrm{~m} / \mathrm{s}^{2}$.

- The acceleration value rises up then falls down in standing up state, and it goes in opposite direction (i.e. falls down then rises up) in sitting down stage. In both states, the difference between the maximum and minimum value is less than $3 \mathrm{~m} / \mathrm{s}^{2}$.

The pattern generated in falling state is somewhat similar to the pattern generated in sitting down state. But the difference between minimum and maximum value is much higher in the former state, around $8 \mathrm{~m} / \mathrm{s}^{2}$ compared to less than $2 \mathrm{~m} / \mathrm{s}^{2}$.

\section{PROPOSED AlgORITHM}

\section{A. Algorithm description}

Based on the above observation, an algorithm for fall detection based on thresholds is developed. Specifically, the following terms is used in the algorithm:

- Detection period: the duration in which acceleration values are extracted and analyzed to determine a fall event. Based on the previous step, detection period is about 1 to 2 seconds.

- Low threshold acceleration: this is the acceleration value that is lower than the acceleration values generated by most activities. In other words, only a fall event can generate acceleration values which are lower than this threshold.

- High threshold acceleration: this value has an opposite meaning with the low threshold acceleration value defined above. It is the acceleration value that is higher than the acceleration values generated by most activities, except the acceleration value generated in a fall.

In a detection period, a fall event is alerted if the following conditions are met:

- There is at least one acceleration value $\mathrm{v} 1$ which is lower than the low threshold acceleration.

- There is at least one acceleration value v2 which is higher than the high threshold acceleration. 
- The time point $\mathrm{t} 1$ when $\mathrm{v} 1$ happens must be preceded the time point $\mathrm{t} 2$ when $\mathrm{v} 2$ happens.

The pseudocode for the above fall detection algorithm is given below:



\section{B. Algorithm refinement}

In the above algorithm, the actual values of low threshold acceleration and high threshold acceleration are the main factors affecting the performance of our proposed fall detection technique. Specifically, the performance of the fall detection technique is evaluated by the 2 following criteria:

- True positive rate: the number of detected falls over the total number of falls. A good system must possess high true positive rate.

- False positive rate: the number of wrongly detected falls over the total number of normal events. We want this number as low as possible.

According to our experiments, achieving a good balancing of the trade-off between true positive rate and false positive rate is challenging. In the fall detection algorithm, if the range between low and high threshold acceleration is set to be broad enough, the false positive rate may achieve at zero percent. However, at that point, the true positive rate is concurrently very low. On the other hand, if the range between low and high threshold is so tight, the true positive rate may reach a perfect score at $100 \%$, but the false positive rate may be too high. This situation is quite dangerous because an individual could have fallen without being detected by the system.

To improve concurrently both true positive rate and false positive rate, an additional checking layer is added after a fall is detected. This check verifies if a person can return to normal activities after a fall is detected. In case the individual does not perform any actions after the suspected fall in about 3 seconds, there is a high possibility that a real fall event has just occurred and caused "long lie" [33]. In the long lie state, the generated acceleration values are stable due to inactivity of the fallen person. As a result, we define several new terms to apply in the long lie detection algorithm:

- long lie period: the duration in which no activities are detected after of a suspected fallen event.

- low long lie threshold acceleration: the acceleration value that is lower than all acceleration values generated during long lie period.

- high long lie threshold acceleration: the acceleration value that is higher than all acceleration values generated during long lie period.

With these terms, the pseudocode for proposed long lie detection algorithm is given below:

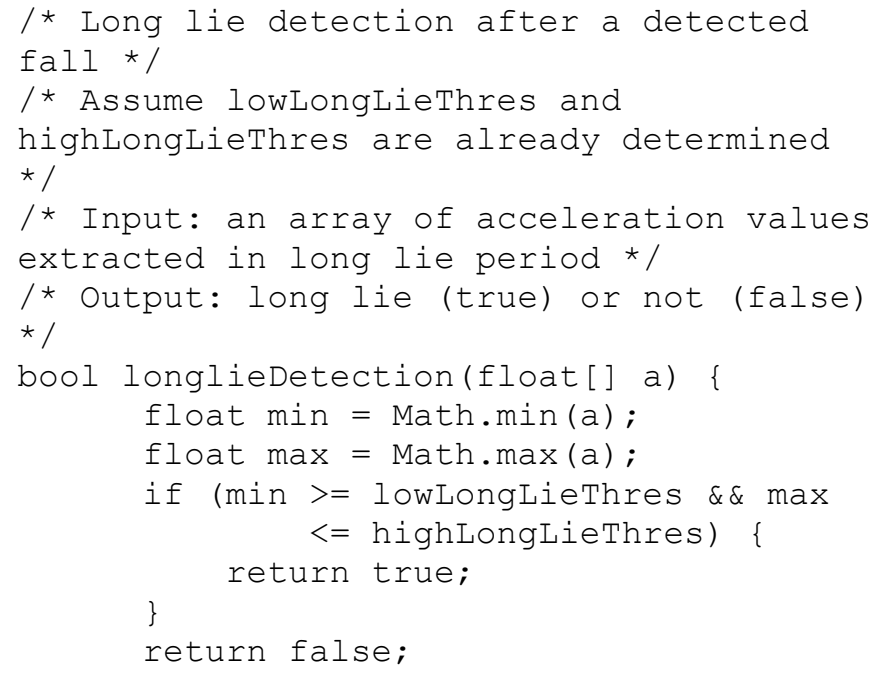

\section{EXPERIMENTS}

\section{A. Application prototype}

To evaluate the performance of the proposed fall detection technique, we develop a working prototype on Android platform and perform the experiment with volunteers. Android is chosen solely because of its popularity. In fact, the detection algorithm is independent of the underlying operating system, therefore could be deployed in any OS.

The components of the prototype are illustrated in Figure 1.

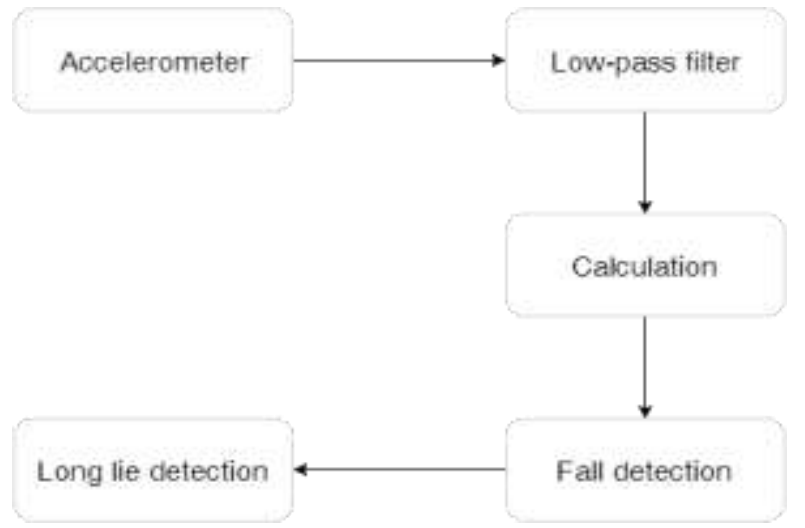

Fig. 1. Components of the working prototype 
As illustrated in Figure 2., the working prototype contains 5 main components. At first, acceleration values are retrieved from mobile accelerometer; these acceleration values then go through a low-pass filter to remove noises. After that, the smoothed output data is utilized to calculate acceleration amplitudes; Finally, fall detection and long lie detection algorithms will determine if a fall event occurs or not based on the calculated amplitudes.

\section{B. Experiment setting}

Five volunteers participate in the experiment to evaluate the performance of the proposed fall detection technique. The group consists 3 men and 2 women from 25 to 35, weights from $50 \mathrm{~kg}$ to $70 \mathrm{~kg}$, and heights from $155 \mathrm{~cm}$ to $170 \mathrm{~cm}$. Although the target users of fall detection system are elderly, it is difficult to invite them into the experiments because of safety requirements. Therefore, our experiment is evaluated on 5 young and healthy individuals.

Each volunteer is requested to put an Android-powered ASUS Zenfone 2 into his/her front pant pocket. This device contains a 5.5-inches screen, an Intel Atom CPU (quad core $2.3 \mathrm{GHz}$ ) and 4GB RAM memory. The device's weight is $170 \mathrm{~g}$ and has built-in Android 5.0. Our application prototype has been installed into the devices before the experiments.

The volunteers are required to perform these following activities twice:

- Stand up from the sitting posture,

- Sit down from the standing posture,

- Walk normally in 5 seconds,

- Fall down onto a soft mattress,

- Lie down in 5 seconds.

The purpose of the first 4 activities is to achieve data to decide the detection period, low detection threshold acceleration, and high detection threshold acceleration. The purpose of the last activity is to determine the long lie period, low long lie threshold acceleration and high long lie acceleration. In the first 4 activities, the volunteers are requested to fall hard enough to be able to classify of other normal activities. In other words, for any individual, if the acceleration generated in a fall cannot be differentiated from the acceleration generated in other activities, we discard all the related data and ask that person to perform these 4 activities again.

\section{Experiment result and discussion}

Beside the fall detection algorithm, long lie detection approach is an enhancement algorithm to confirm the suspicious fall event. For that reason, we can tolerate a little bit high false positive rate to compensate for a higher true positive rate. Therefore, for each volunteer, the higher value between 2 low detection threshold accelerations attained in 2 falls is chosen. The lower value between 2 high detection threshold acceleration values attained in 2 falls is chosen also. Similarly, the detection period for each individual is chosen as the longer one between the 2 periods attained in experiments.
The long lie period is set to 3 seconds. The low long lie threshold acceleration and high long lie threshold acceleration for each person are set to the minimum and maximum acceleration values attained during 10 seconds (we combine 2 lying down experiments together) lying down respectively.

Table 1 displays the fall and long lie detection algorithms' parameters after the experiments:

\section{TABLE I. FALL AND LONG LIE DETECTION PARAMETERS}

\begin{tabular}{|l|l|l|l|l|l|}
\hline ID & $\begin{array}{l}\text { Low } \\
\text { detection } \\
\text { threshold } \\
\text { accelerati- } \\
\text { on }\left(\mathrm{m} / \mathrm{s}^{2}\right)\end{array}$ & $\begin{array}{l}\text { High } \\
\text { detection } \\
\text { threshold } \\
\text { accelerati- } \\
\text { on }\left(\mathrm{m} / \mathrm{s}^{2}\right)\end{array}$ & $\begin{array}{l}\text { Detecti- } \\
\text { on } \\
\text { period } \\
(\mathrm{s})\end{array}$ & $\begin{array}{l}\text { Low long } \\
\text { lie } \\
\text { threshold } \\
\text { acceleratio } \\
\mathrm{n}\left(\mathrm{m} / \mathrm{s}^{2}\right)\end{array}$ & $\begin{array}{l}\text { High long } \\
\text { lie } \\
\text { threshold } \\
\text { acceleratio } \\
\mathrm{n}\left(\mathrm{m} / \mathrm{s}^{2}\right)\end{array}$ \\
\hline 1 & 8.4 & 17.3 & 1.6 & 9.6 & 9.9 \\
\hline 2 & 8.0 & 16.5 & 2.2 & 9.7 & 9.9 \\
\hline 3 & 8.3 & 16.8 & 2.0 & 9.7 & 10.0 \\
\hline 4 & 7.5 & 15.6 & 1.8 & 9.7 & 10.1 \\
\hline 5 & 8.2 & 16.2 & 1.6 & 9.6 & 10.0 \\
\hline
\end{tabular}

After collecting all the fall detection and long lie detection parameters, the 2 proposed detection algorithms are modified to utilize these parameters for decision making. Then, each volunteer is asked to use the application for an extended period of time, i.e. about 2 minutes. During that time, each volunteer is requested to either walking, running, or falling. Each person needs to fall at least 5 times, and stays lying down for at least 5 seconds after each fall. The result of these experiments is given in Table 2.

TABLE II. RESUlt WHEN WSING THE APP FOR AN EXTENDED PERIOD

\begin{tabular}{|l|l|l|l|l|}
\hline Person & $\begin{array}{l}\text { Number of } \\
\text { falls }\end{array}$ & $\begin{array}{l}\text { Number of } \\
\text { detected falls }\end{array}$ & $\begin{array}{l}\text { Number of } \\
\text { false positives } \\
\text { without long } \\
\text { lie check }\end{array}$ & $\begin{array}{l}\text { Number of } \\
\text { false positives } \\
\text { with long lie } \\
\text { check }\end{array}$ \\
\hline 1 & 5 & 5 & 5 & 1 \\
\hline 2 & 5 & 4 & 3 & 0 \\
\hline 3 & 6 & 6 & 0 & 0 \\
\hline 4 & 5 & 5 & 0 & 0 \\
\hline 5 & 6 & 4 & 3 & 0 \\
\hline
\end{tabular}

The data in Table 2 shows that our proposed technique achieves the true detection rate of $93 \%$. Without long lie check, total of 11 false alarms occurs, whereas this measurement reduces to 1 if the long lie check is integrated. In our experiments, all false positives are created during quick running and they come together, not as separated ones. When long lie detection is added, most of the fall positive cases are eliminated. That exceptional case is caused by one person' standing still after running due to his tiredness.

\section{CONCLUSIONS AND FUTURE WORKS}

In this paper, we have proposed a technique for automatic fall detection using smartphones. The approach utilizes the phone accelerometer's captured data to make decision. The proposed technique consists of 2 algorithms: fall detection and long lie detection. The former is used to check the occurrence of a fall, while the latter is used to find out if there is a lying down state after that fall. By combining these 2 algorithms, we can partly solve the trade-off issue between achieving a high true positive rate and keeping a low false positive rate. 
The proposed technique is then implemented as an Android application for experiment. Due to safety constraints, only young and healthy people are invited to participate. They are asked to perform intentional falls as well as other normal activities. The data generated during these actions are used to get detection parameters. These parameters are then applied in detection algorithms to evaluate the application performance for an extended period. Experiment results show the potential of using this application for fall detection when achieving a true positive rate of $93 \%$ and only generates 1 false positive during 10 minutes experiment of 5 volunteer people.

There are still some drawbacks that cannot be completely solved by the algorithm. The first one is not able to organize a realistic environment for experiments. Because falls may cause serious injuries, the experiment 's volunteers just consist of young and healthy individuals, who are particularly not the main target of the fall detection technique. This may make the parameters learnt in experiments not match with the practical conditions. Furthermore, volunteers only fall onto a soft surface during evaluation which makes the result may be very different when comparing with real falls onto hard surfaces.

Another problem is the limitation in the extensive experiments with large number of volunteers. Due to time and cost constraints, we do not have many people to join our experiments which may cause the achieved results not representative enough. In addition, the activities performed are insufficient which do not produce all possible acceleration patterns in reality. For example, we do not examine strong activities such as jumping, which may create similar acceleration patterns falling. By experimenting more with such activities, we can fine-tune the proposed algorithms and make our application more suitable in real-world utilization.

\section{ACKNOWLEDGMENT}

This research is funded by Vietnam National University HoChiMinh City (VNU-HCM) under grant number C2015-2006

\section{REFERENCES}

[1] Department of Economic and Social Affairs United Nations, 2002. World population ageing: 1950-2050. UN.

[2] Centers for Disease Control and Prevention. Important Facts about Falls. Available

http://www.cdc.gov/homeandrecreationalsafety/falls/adultfalls.html (accessed 3/2016)

[3] Alexander, B.H., Rivara, F.P. and Wolf, M.E., 1992. The cost and frequency of hospitalization for fall-related injuries in older adults. American journal of public health, 82(7), pp.1020-1023.

[4] Sterling, D.A., O'Connor, J.A. and Bonadies, J., 2001. Geriatric falls: injury severity is high and disproportionate to mechanism. Journal of Trauma and Acute Care Surgery, 50(1), pp.116-119.

[5] Stevens, J.A., Corso, P.S., Finkelstein, E.A. and Miller, T.R., 2006. The costs of fatal and non-fatal falls among older adults. Injury prevention, 12(5), pp.290-295.

[6] Igual, R., Medrano, C. and Plaza, I., 2013. Challenges, issues and trends in fall detection systems. Biomedical engineering online, 12(1), p.1.

[7] Gibson, M.J., Andres, R.O., Isaacs, B., Radebaugh, T. and Wormpetersen, J., 1987. The Prevention of Falls in Later Life-A Report of the Kellogg-International-Work-Group on the Prevention of Falls by the Elderly. Danish Medical Bulletin, 34, pp.1-24.

[8] Friedman, S.M., Munoz, B., West, S.K., BandeenRoche, K. and Fried, L.P., 1997, September. Falls and fear of falling: Which comes first? In
JOURNAL OF THE AMERICAN GERIATRICS SOCIETY (Vol. 45, No. 9, pp. P186-P186). 351 WEST CAMDEN ST, BALTIMORE, MD 21201-2436: WILLIAMS \& WILKINS.

[9] Brownsell, S. and Hawley, M.S., 2004. Automatic fall detectors and the fear of falling. Journal of telemedicine and telecare, 10(5), pp.262-266.

[10] Rubenstein, L.Z. and Josephson, K.R., 2002. The epidemiology of falls and syncope. Clinics in geriatric medicine, 18(2), pp.141-158.

[11] Tinetti, M.E., Liu, W.L. and Claus, E.B., 1993. Predictors and prognosis of inability to get up after falls among elderly persons. Jama, 269(1), pp.65-70.

[12] Lord, S.R., Sherrington, C., Menz, H.B. and Close, J.C., 2007. Falls in older people: risk factors and strategies for prevention. Cambridge University Press.

[13] Noury, N., Fleury, A., Rumeau, P., Bourke, A.K., Laighin, G.O., Rialle, V. and Lundy, J.E., 2007, August. Fall detection-principles and methods. In 2007 29th Annual International Conference of the IEEE Engineering in Medicine and Biology Society (pp. 1663-1666). IEEE.

[14] Bagalà, F., Becker, C., Cappello, A., Chiari, L., Aminian, K., Hausdorff, J.M., Zijlstra, W. and Klenk, J., 2012. Evaluation of accelerometerbased fall detection algorithms on real-world falls. PloS one, 7(5), p.e37062.

[15] Miaou, S.G., Sung, P.H. and Huang, C.Y., 2006, April. A customized human fall detection system using omni-camera images and personal information. In 1 st Transdisciplinary Conference on Distributed Diagnosis and Home Healthcare, 2006. D2H2. (pp. 39-42). IEEE.

[16] Fu, Z., Delbruck, T., Lichtsteiner, P. and Culurciello, E., 2008. An address-event fall detector for assisted living applications. IEEE transactions on biomedical circuits and systems, 2(2), pp.88-96.

[17] Hazelhoff, L. and Han, J., 2008, October. Video-based fall detection in the home using principal component analysis. In International Conference on Advanced Concepts for Intelligent Vision Systems (pp. 298-309). Springer Berlin Heidelberg.

[18] Cucchiara, R., Prati, A. and Vezzani, R., 2007. A multi-camera vision system for fall detection and alarm generation. Expert Systems, 24(5), pp.334-345.

[19] Hazelhoff, L. and Han, J., 2008, October. Video-based fall detection in the home using principal component analysis. In International Conference on Advanced Concepts for Intelligent Vision Systems (pp. 298-309). Springer Berlin Heidelberg.

[20] Liu, C.L., Lee, C.H. and Lin, P.M., 2010. A fall detection system using k-nearest neighbor classifier. Expert systems with applications, 37(10), pp.7174-7181.

[21] Vishwakarma, V., Mandal, C. and Sural, S., 2007, December. Automatic detection of human fall in video. In International conference on pattern recognition and machine intelligence (pp. 616-623). Springer Berlin Heidelberg.

[22] Rimminen, H., Lindström, J., Linnavuo, M. and Sepponen, R., 2010. Detection of falls among the elderly by a floor sensor using the electric near field. IEEE transactions on information technology in biomedicine: a publication of the IEEE Engineering in Medicine and Biology Society, 14(6), pp.1475-1476.

[23] Cucchiara, R., Prati, A. and Vezzani, R., 2007. A multi-camera vision system for fall detection and alarm generation. Expert Systems, 24(5), pp.334-345.

[24] Miaou, S.G., Sung, P.H. and Huang, C.Y., 2006, April. A customized human fall detection system using omni-camera images and personal information. In 1st Transdisciplinary Conference on Distributed Diagnosis and Home Healthcare, 2006. D2H2. (pp. 39-42). IEEE.

[25] Anderson, D., Luke, R.H., Keller, J.M., Skubic, M., Rantz, M. and Aud, M., 2009. Linguistic summarization of video for fall detection using voxel person and fuzzy logic. Computer vision and image understanding, 113(1), pp.80-89.

[26] Doukas, C., Maglogiannis, I., Tragas, P., Liapis, D. and Yovanof, G., 2007, September. Patient fall detection using support vector machines. In IFIP International Conference on Artificial Intelligence Applications and Innovations (pp. 147-156). Springer US.

[27] Cheng, J., Chen, X. and Shen, M., 2013. A framework for daily activity monitoring and fall detection based on surface electromyography and 
accelerometer signals. IEEE journal of biomedical and health informatics, 17(1), pp.38-45.

[28] Yuwono, M., Moulton, B.D., Su, S.W., Celler, B.G. and Nguyen, H.T., 2012. Unsupervised machine-learning method for improving the performance of ambulatory fall-detection systems. Biomedical engineering online, 11(1), p.1.

[29] Kerdegari, H., Samsudin, K., Ramli, A.R. and Mokaram, S., 2012, June. Evaluation of fall detection classification approaches. In Intelligent and Advanced Systems (ICIAS), 2012 4th International Conference on (Vol. 1, pp. 131-136). IEEE.

[30] Sposaro, F. and Tyson, G., 2009, September. iFall: an Android application for fall monitoring and response. In 2009 Annual
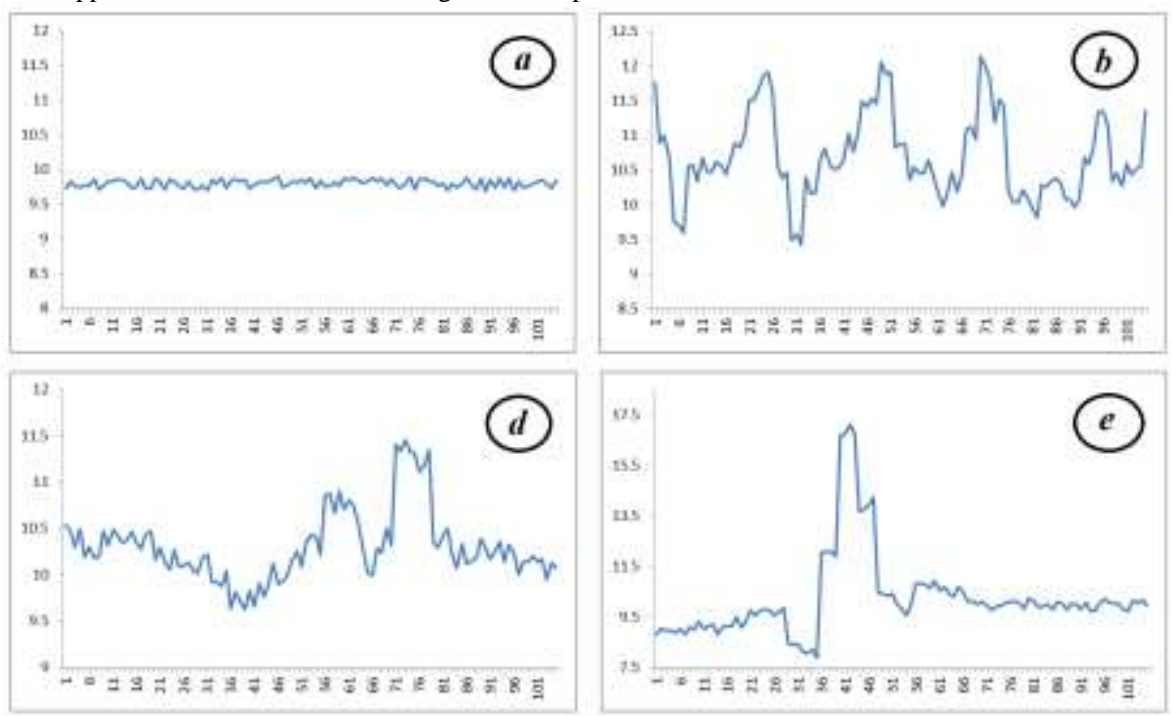

International Conference of the IEEE Engineering in Medicine and Biology Society (pp. 6119-6122). IEEE.

[31] Lee, R.Y. and Carlisle, A.J., 2011. Detection of falls using accelerometers and mobile phone technology. Age and ageing, p.afr050.

[32] Fang, S.H., Liang, Y.C. and Chiu, K.M., 2012, January. Developing a mobile phone-based fall detection system on android platform. In Computing, Communications and Applications Conference (ComComAp), 2012 (pp. 143-146). IEEE.

[33] Nevitt, M.C., Cummings, S.R. and Hudes, E.S., 1991. Risk factors for injurious falls: a prospective study. Journal of gerontology, 46(5), pp.M164-M170.

Fig. 2. Plotting of acceleration values and patterns in various activities

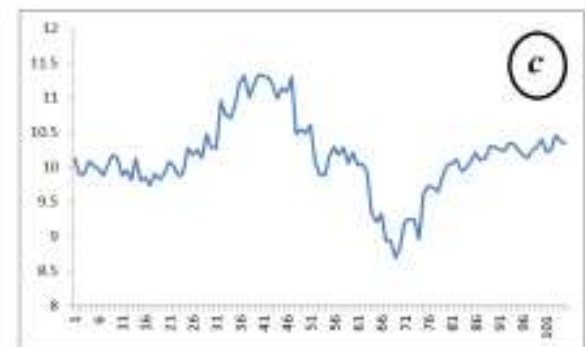

Acceleration values and patterns generated in various activities:
a. Sitting
b. Walking
c. Standing up
d. Sitting down
e. Falling down 